\title{
Monodispersed and size-controlled multibranched gold nanoparticles with nanoscale tuning of surface morphology $\dagger$
}

\author{
Gabriele Maiorano, ${ }^{a}$ Loris Rizzello, ${ }^{a}$ Maria Ada Malvindi, ${ }^{a}$ Sangaru Shiv Shankar, ${ }^{a}$ Luigi Martiradonna, \\ Andrea Falqui, ${ }^{b}$ Roberto Cingolani ${ }^{a b}$ and Pier Paolo Pompa ${ }^{* a}$
}

\author{
Received 26th January 2011, Accepted 2nd March 2011 \\ DOI: $10.1039 / c 1 n r 10107 b$
}

\begin{abstract}
A novel seed-mediated synthetic route to produce multibranched gold nanoparticles is reported, in which it is possible to precisely tune both their size and nanostructuration, while maintaining an accurate level of monodispersion. The nanoscale control of surface nanoroughness/branching, ranging from small bud-like features to elongated spikes, allows to obtain fine tuning of the nanoparticle optical properties, up to the red and near-IR region of the spectrum. Such anisotropic nanostructures were demonstrated to be excellent candidates for SERS applications, showing significantly higher signals with respect to the standard spherical nanoparticles.
\end{abstract}

\section{Introduction}

The increasing understanding of the size- and shape-dependent properties of nanomaterials results in new ideas and novel applications. $^{1}$ As such, there is an increasing demand for protocols delivering nanomaterials of good quality and high control of the final product. Among various nanomaterials, gold nanoparticles (AuNPs) are of particular interest because of their multiple biological applications and surface functionalization possibilities in combination with their size-, shape- and surfacedependent properties. ${ }^{2-4}$ To date, different shapes of AuNPs have been synthesized, namely nanorods, ${ }^{5}$ nanotriangles, ${ }^{6}$ nanocubes/ nanocages, ${ }^{7}$ and nanoshells. ${ }^{8}$ Recently, multibranched gold nanoparticles are attracting interest, due to their catalytic activity, ${ }^{3}$ molecular detection ${ }^{9,10}$ and biological applications, such as in biosensing, ${ }^{11}$ immunoassays ${ }^{12}$ and dark field imaging of cells. ${ }^{13}$ These nanoparticles have also been alternatively named by different groups as nanoflowers, ${ }^{14}$ nanostars ${ }^{15}$ or nanourchins. ${ }^{16}$ Similar to other anisotropic nanoparticles discussed above, they exhibit interesting optical properties, depending on their structural features. ${ }^{14-19}$ The multibranched nanoparticles are, thus, promising candidates to meet the increasing demands of stable, multifunctional nanoparticles for biodiagnostics and nanomedicine. Additionally, these anisotropic nanostructures exhibit strong electromagnetic field enhancement upon irradiation and, for this reason, they are important for Surface

${ }^{a}$ Italian institute of Technology, Center for Biomolecular Nanotechnology (CBN), Via Barsanti 1, 73010 Arnesano, LE, Italy. E-mail: pierpaolo. pompa@iit.it; Fax: +39-0832-295708; Tel: +39-0832-295714

${ }^{b}$ Italian Institute of Technology, Central Research Laboratories, Via Morego, 30-16136 Genova, Italy

$\uparrow$ Electronic supplementary information (ESI) available. See DOI: $10.1039 / \mathrm{c} 1 \mathrm{nr} 10107 \mathrm{~b}$
Enhanced Raman Scattering (SERS) spectroscopy and imaging., 9,20

Most reported protocols to synthesize branched gold nanoparticles employ different surfactants, polymers or biomolecules as shape-directing agents in seed mediated $^{15,21}$ or direct synthesis. ${ }^{22}$ However, all these interesting approaches have some drawbacks, such as the long reaction times or the large size and shape dispersion. Moreover, the shape-directing agents (cetyltrimethylammonium bromide, CTAB; polyvinylpyrrolidone, PVP; etc.) can strongly adsorb onto the nanoparticles surface, severely restricting their application fields, as they are difficult to remove or modify. In some selected reports, alternative approaches that do not use such strong surface adsorbing molecules have been demonstrated. ${ }^{23,24}$ All the above mentioned strategies were able to produce multibranched AuNPs and partially control, in certain cases, their branching. However, to the best of our knowledge, the control of both size and surface nanoroughness/branching of these particles using a single synthesis approach has never been accomplished and still remains a challenge.

In this paper we demonstrate a synthetic route toward multibranched gold nanoparticles (Au MBNPs) fabrication that allows to finely control both surface nanoroughness/branching and final size in a reproducible way. Our synthesis procedure is surfactantless and high-yield, and it is based on the enlargement of colloidal AuNPs (seeds) by using the Au surface-catalyzed reduction of $\mathrm{Au}^{3+}$ by hydroxylamine $\left(\mathrm{NH}_{2} \mathrm{OH}\right){ }^{25,26}$ This process, usually exploited for size control of spherical nanoparticles, was coupled with the ability of 2-[4-(2-hydroxyethyl)-1-piperazinyl] ethane-sulfonic acid (HEPES) to act as a precise shape-directing agent. HEPES is a popular $\mathrm{pH}$ buffer, used extensively in chemistry and biochemistry laboratories and in tissue culturing. ${ }^{27}$ Recently, HEPES was employed for a single step synthesis of branched colloidal gold nanoflowers, in which it acted as both 
a weakly reducing and a shape-directing agent. ${ }^{14,28}$ However, fine tuning of the optical properties, by controlling their size or the uniform level of branching, was not demonstrated. In our seed mediated synthesis, HEPES is predominantly employed as a shape-directing agent, and it is exploited efficiently to control the branching and size of the gold nanorough particles, whereas hydroxylamine operates as the reducing agent. This technique yields high quality nanoparticles without any purification step, and the branching on a spherical core can be precisely controlled at nanometre level, from small bud-like features to elongated spikes. Such a remarkable control gives the opportunity to conveniently tune their optical properties over a wide range, up to the red and IR region of the spectrum. Furthermore, these nanoparticles were demonstrated to act as optimal nanostructures for SERS applications if compared to the classical spherical NPs, providing both significantly higher Raman signals and the possibility to use a very low concentration of the reporter molecules.

\section{Results and discussion}

Monodisperse gold nanoparticles of controlled size can be synthesized by the method reported by Brown and $\mathrm{Natan}^{26}$ and used for the growth of different multibranched nanoparticles. Here, we exploited, as seeds, 18 and $40 \mathrm{~nm}$ spherical, citratecapped AuNPs (see Experimental section and Fig. S1 $\uparrow$ in the ESI). In order to modulate the surface structure at the nanoscale with different levels of branching, the Au seeds were grown in the presence of increasing amount of HEPES, ranging from 0 to 0.2 , $1,2.5,5$ up to $25 \mathrm{mM}$ concentration in the final solution (see Experimental section). The amount of gold ions used in all reactions was equivalent to that required for the growth of a continuous gold layer of $\sim 10 \mathrm{~nm}$ thickness around the seeds, to achieve a final size of $\sim 40$ and $\sim 60 \mathrm{~nm}$ (starting from seeds of 18 and $40 \mathrm{~nm}$, respectively). The growth reaction occurred instantaneously during the dropwise addition of chloroauric acid to a seed solution containing hydroxylamine as a reducing agent.

In Fig. 1, we report representative TEM images of the gold nanoparticles that were formed under different conditions. At first glance, very good control of the final sizes is accomplished, leading to highly monodisperse samples in all cases. Furthermore, a very precise control of the nanostructuration of the gold nanoparticles in each set of samples is also obtained. In particular, a clear modulation of the surface nanostructuration can be noticed, ranging from very low levels of nanoroughness with bud-like features of $\sim 4 \mathrm{~nm}$ (Fig. 1c and d), to highly rough Au MBNPs with branches up to $\sim 20 \mathrm{~nm}$ (Fig. $1 \mathrm{~m}$ and $\mathrm{n}$ ). The extent of surface nanostructuration roughly follows the same trend for both $40 \mathrm{~nm}$ (left column of Fig. 1) and $60 \mathrm{~nm}$ Au MBNPs (right column of Fig. 1). At the same time, the size distribution data of the MBNPs, listed in Table 1 (see also Fig. S2 and S $3 \uparrow$ ), revealed that we were able to efficiently control also their final size. The low magnification SEM images (Fig. 2) further confirmed the excellent size dispersion of the nanoparticles, around $6 \pm 2 \%$ for both sizes of MBNPs (see also Fig. S2 and S3†). In addition, Fig. 1 shows that the branching is very similar for the two sizes, while the number of branches is higher in the case of bigger nanoparticles, owing to the larger surface area of the seed nanoparticles. Finally, we remark that, in the absence of HEPES,

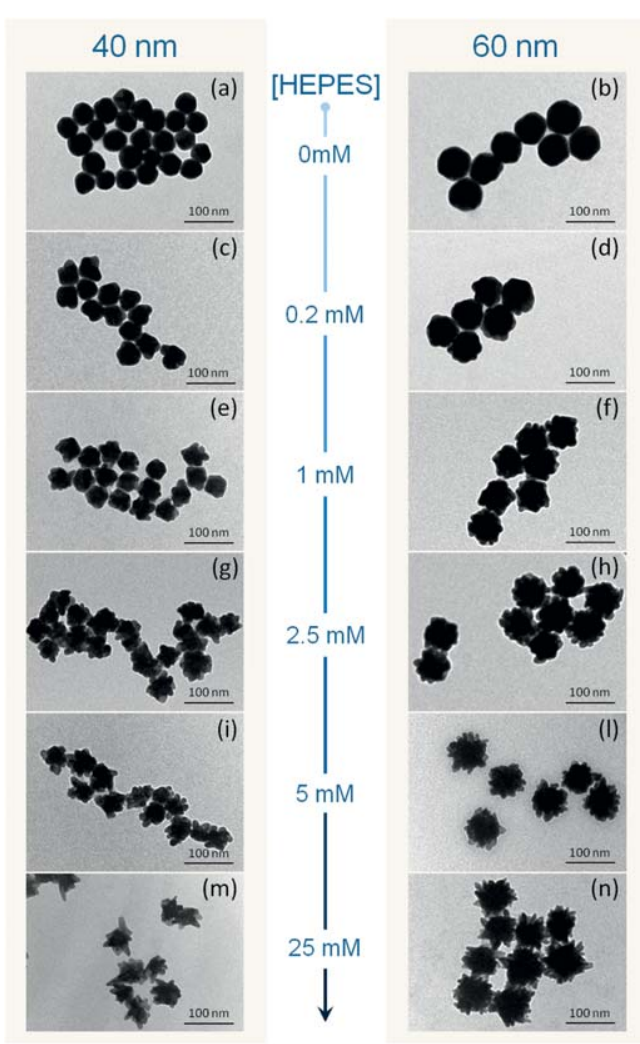

Fig. 1 TEM images of 40 and $60 \mathrm{~nm} \mathrm{Au} \mathrm{MBNPs} \mathrm{synthesized} \mathrm{in}$ increasing concentration of HEPES ranging from $0 \mathrm{mM}$ HEPES ( $a$ and $b$ ) to $25 \mathrm{mM}$ HEPES ( $\mathrm{m}$ and $\mathrm{n}$ ).

the growth of seeds only leads to enlarged spherical nanoparticles without surface structuration. This indicates the importance of HEPES as the shape-directing agent in the present synthesis procedure.

The precise control of the nanostructuration of the AuNPs can also be appreciated by comparing their optical properties. Fig. 3a and $\mathrm{b}$ show the UV-Vis spectra of the 18 and $40 \mathrm{~nm}$ gold nanoparticle seeds grown in the presence of different concentrations of HEPES, as mentioned above. An increasing red-shift of the surface plasmon resonance (SPR) peak occurs as a function of the increasing nanostructuration of the MBNPs up to the NIR region (peaking at $c a .720 \mathrm{~nm}$ for the $40 \mathrm{~nm}$ MBNPs and at

Table 1 Mean size and standard deviation (SD) for 40 and $60 \mathrm{~nm} \mathrm{Au}$ MBNPs (taken from at least 100 NPs)

\begin{tabular}{llll}
\hline & [HEPES]/mM & Mean diameter/nm & SD/nm \\
\hline 40 nm Au MBNPs & 0 & 40.6 & 2.3 \\
& 0.2 & 40.4 & 3.0 \\
& 1 & 40.3 & 2.8 \\
& 2.5 & 40.0 & 2.6 \\
$60 \mathrm{~nm} \mathrm{Au} \mathrm{MBNPs}$ & 5 & 40.5 & 3.4 \\
& 25 & 40.4 & 3.2 \\
& 0.2 & 59.8 & 3.5 \\
& 1 & 59.9 & 4.1 \\
& 2.5 & 60.0 & 3.6 \\
& 5 & 60.5 & 3.6 \\
& 25 & 60.3 & 3.1 \\
& & 60.1 & 5.0 \\
\hline
\end{tabular}



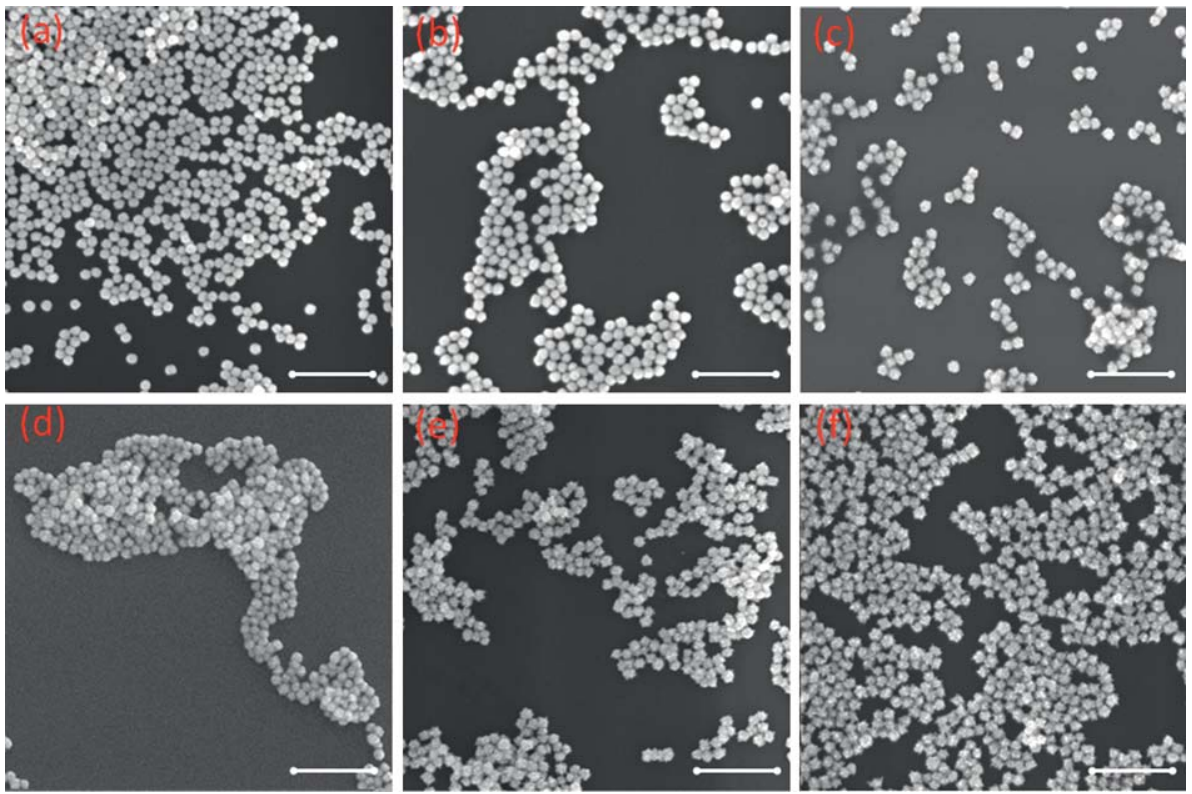

Fig. 2 Representative SEM images of $60 \mathrm{~nm}$ Au MBNPs obtained at different HEPES concentrations, namely $0 \mathrm{mM}$ (a), $0.2 \mathrm{mM}$ (b), $1 \mathrm{mM}$ (c), $2.5 \mathrm{mM}$ (d), $5 \mathrm{mM}$ (e) and $25 \mathrm{mM}$ (f), highlighting the high monodispersion of such NPs. Scale bars are $500 \mathrm{~nm}$.

ca. $708 \mathrm{~nm}$ for the $60 \mathrm{~nm}$ MBNPs). In the case of nanoparticles grown in the presence of a low concentration of HEPES, the SPR bands merely show a small red-shift in the peak value. On the other hand, nanoparticles with higher nanostructuration exhibit also the presence of a new absorption band. By analyzing these data in combination with the TEM images (Fig. 1), it can be inferred that the less rough MBNPs with small bud-like surface features are characterized by an absorption spectrum similar to a spherical nanoparticle, with a red shift proportional to the increased dimension. ${ }^{4}$ In the presence of a higher concentration of HEPES, when these bud-like structures outgrow into branch-like structures with higher aspect ratios, an additional band resembling the longitudinal plasmon peak of gold nanorods appears at longer wavelengths. ${ }^{29} \mathrm{With}$ increasing the aspect ratio of the branches, the longitudinal component of the plasmon band becomes more and more intense and red-shifts. Concurrently, above $2.5 \mathrm{mM}$ HEPES concentration, the transversal component of the plasmon band begins to appear as a shoulder blue shifted with respect to the single plasmon peaks observed for the Au MBNPs with lower branching. These features are similar to the optical characteristics that have been demonstrated experimentally and predicted theoretically for gold nanorods. ${ }^{29}$
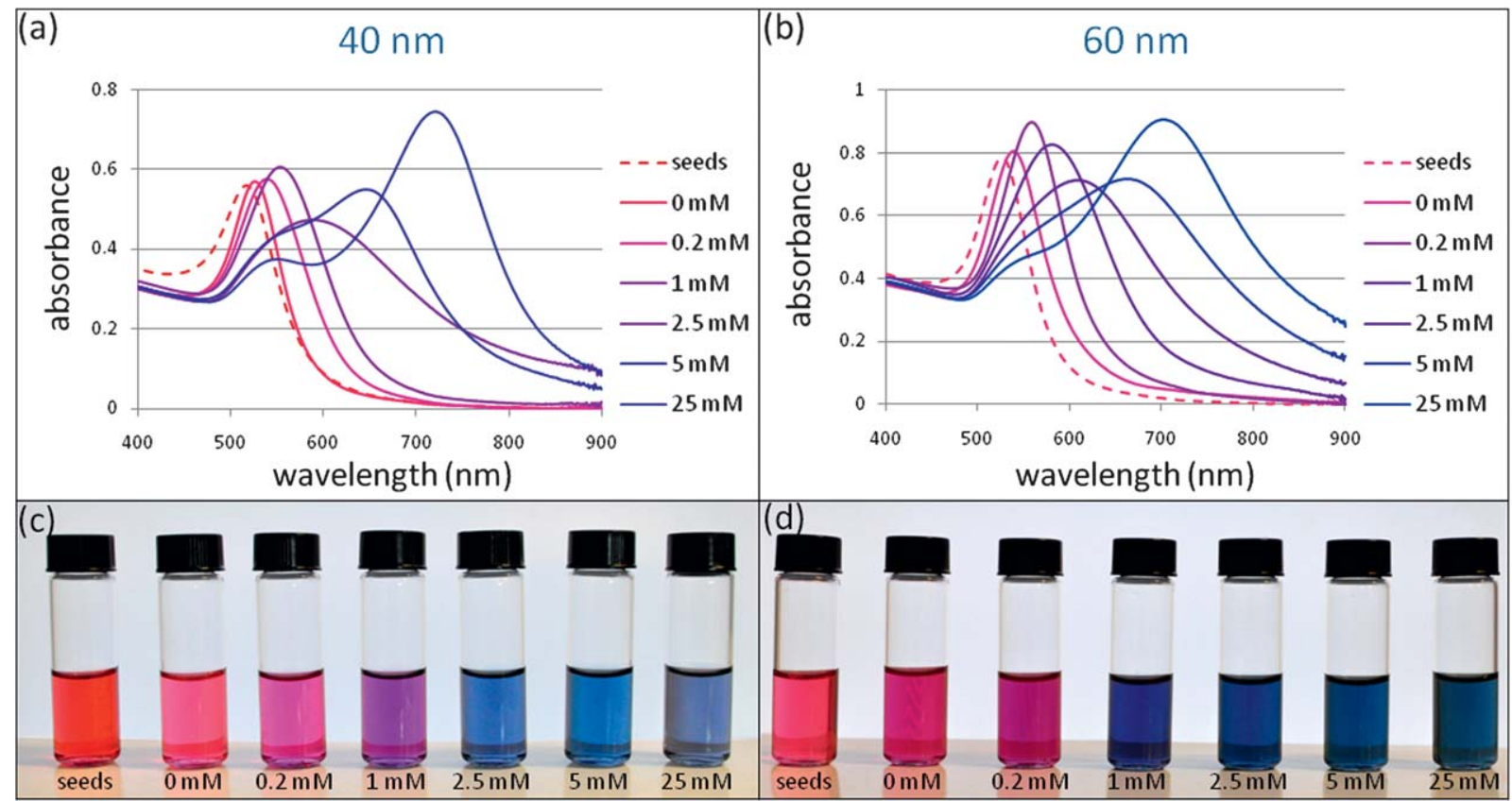

Fig. 3 (a and b) UV-Vis spectra of the 40 and $60 \mathrm{~nm}$ Au MBNPs grown in the presence of different concentrations of HEPES. The increasing SPR redshift is also well observable by a representative photograph of freshly synthesized Au MBNPs (c and d). 
Such optical behavior can be clearly noticed even with the naked eye (Fig. $3 \mathrm{c}$ and $\mathrm{d}$ ) by the changing color of the sample solutions (from red to blue-grey). The small difference observed between the 40 and $60 \mathrm{~nm}$ MBNPs (maximum red shift of $720 \mathrm{~nm}$ and $708 \mathrm{~nm}$, respectively) is attributed to the relatively smaller size of $40 \mathrm{~nm}$ MBNPs core with respect to the branches, leading to a more distinct longitudinal plasmon peak with a larger redshift. ${ }^{15,30}$ These results demonstrate that our new synthetic route, combining the ability of hydroxylamine sulfate to act as a reducing agent with the capability of HEPES to operate as a shape-directing mediator, allows us to obtain highly controlled Au MBNPs, in terms of both size and surface nanostructuration, thus presenting an efficient way to finely tune their optical properties.

In order to validate that, in this synthetic route, HEPES acts predominantly as the shape directing agent for Au MBNPs, with a negligible role as reducing agent, we carried out also synthesis in the absence of hydroxylamine sulfate in the reaction mixture (negative control). The time dependent evolution of such reactions, monitored by UV-Vis measurements, elucidated that the growth of Au MBNPs in the absence of hydroxylamine takes a relatively longer time scale, and spectra are not comparable to those observed for the growth with $\mathrm{NH}_{2} \mathrm{OH}$ (Fig. S4†). TEM analyses of these AuNPs revealed the formation of polydisperse NPs with various shapes, due to disparate and uncontrollable phenomena of gold salt reduction (Fig. S5 $\dagger$ ). These latter images show, in fact, the coexistence of both small and bigger multishaped particles, demonstrating the presence of seed-mediated and independently nucleated AuNPs. Concerning our synthetic route, it is important to consider three arguments: (i) HEPES is a slow reducing agent; ${ }^{28}$ (ii) unlike HEPES, hydroxylamine strongly reduces $\mathrm{Au}^{3+}$ ions only in the presence of gold surfaces, ${ }^{25,26}$ (iii) in the direct reaction of HEPES with $\mathrm{Au}^{3+}$ ions, initially, individual nanocrystals are formed, and then they agglomerate and grow anisotropically to form the resultant $\mathrm{Au}$ nanoflowers. ${ }^{14}$ Considering such characteristics, we believe that, in our case, the growth of Au MBNPs does not proceed through the aggregation and growth mechanism, but selectively through directional growth on the seed particle surface, facilitated by hydroxylamine. As hydroxylamine plays the crucial role of surface catalyzed reduction of $\mathrm{Au}^{3+}$ ions, it consumes all the ions prior to the possible formation of independent nanoparticles by the shape directing HEPES molecules. Thus, hydroxylamine, by altering the mechanism of growth, leads to the formation of better monodisperse gold MBNPs. Furthermore, as the growth is now not dependent on the aggregation of individual spherical nanocrystals, the variation of the MBNPs spectral features is not restricted to a range moderately greater than that for spherical particles. ${ }^{14,29}$ As shown above, directed growth on the seed particles allowed us to grow branches of longer dimension, thus tuning their optical properties over a wider range.

To assess the internal structure of the Au MBNPs, we performed high resolution transmission electron microscopy (HRTEM) analyses. From HRTEM measurements, reported in Fig. 4, it is clear that branches are defect free single crystalline. No twin boundaries along the branches could be detected (Fig. 4a), as it was shown in a few other reports. ${ }^{31}$ Therefore, it appears that, in the present case, the branches on the gold nanoparticles do not grow by the addition of gold ions over the
$\{111\}$ faces on either side of the twin boundaries of the seed nanocrystal. Such a mechanism leads to a growth of the branch normal to the twin boundary, away from the surface, resulting in a twin plane running through the central axis of the branch. ${ }^{31}$ On a careful observation of the HRTEM images, it can be noticed that, though there is no twin plane running through the branch, there appears to be a twin plane at the point of attachment of the branch to the core nanoparticle (Fig. 4b). It can be concluded, therefore, that the branches are not growing along the twin boundaries, but they are growing over the adjacent facets bound by the twin boundaries on the seed gold nanoparticles. ${ }^{32} \mathrm{We}$ ascribe such differences in the growth mechanism to the presence of HEPES as the surface binding agent. In fact, in the absence of any strong surface capping agent, the reduced metal ions tend to deposit around the twin boundaries because of their higher interfacial energy that makes them more active. However, when HEPES molecules are added to the solution, they preferably bind more strongly at these active twin boundary regions. Hence, in the presence of appropriate rate of influx of reduced gold atoms, growth of branches appears to preferentially occur over the facets enclosed within the twin boundaries (Fig. 4c). In Fig. 4c, a nascent $\mathrm{Au}$ MBNP synthesized at low concentrations of HEPES is shown. Clearly, the nanoparticles are highly twinned and the raised features within the twin bound areas (marked by black arrows) strongly suggest the preferred growth direction in appropriate conditions.

The highly controlled morphology of our Au MBNPs allowed us to investigate the role that surface nanoroughness, apart from size, exerts over SERS efficiency. SERS spectroscopy is attracting increasing interest for chemical and bioanalytical sensing and imaging applications, ${ }^{33-36}$ and anisotropic metallic NPs were recently exploited as promising SERS substrates; in fact, several studies suggested that these kinds of nanostructures exhibit strong SERS enhancing activity. ${ }^{14,20,37-39}$ The as synthesized 60 $\mathrm{nm}$ Au MBNPs were investigated to characterize their ability to enhance the Raman signal of Crystal Violet (CV) molecules. We focused our attention on $60 \mathrm{~nm}$ Au MBNPs because Krug and co-workers demonstrated that spherical gold nanoparticles with a diameter between 60 and $80 \mathrm{~nm}$ are particularly efficient for SERS, with excitations in the region of red (630-650 nm) and near-infrared $(785 \mathrm{~nm}){ }^{38}$ Three aspects were carefully taken into account in our experiments: (i) working with nanoparticles at the same concentration and surface chemistry; (ii) avoid particles aggregation, which leads to localized formation of extremely high electromagnetic field enhancements, usually called

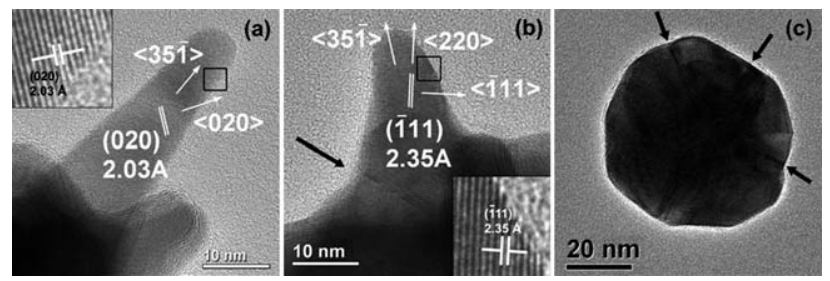

Fig. 4 HRTEM images of Au MBNPs grown in the presence of $5 \mathrm{mM}$ HEPES (a and b) and 0.2 mM HEPES (c). The well grown branches in (a) and (b) are single crystalline with their crystallographic orientations indicated, and the lattice spacing matching with the standard fcc gold values. The black arrows in (b) and (c) indicate the twin boundaries of the nanoparticles. 
hotspots; ${ }^{40}$ (iii) checking for the optimal concentration of the Raman reporter in order to acquire the maximum Raman enhancement. Regarding such latter point, it must be taken into account that the signal intensity is typically weak at low reporter concentrations, it increases reaching a maximum at a proper concentration, while further reporter addition decreases the SERS signal. This behavior is due to the NPs surface coverage by the analyte; above the saturation point, a multilayer of reporter molecules occurs, resulting in a decrease of the SERS signal intensity. ${ }^{9}$

The $60 \mathrm{~nm}$ Au MBNPs series was negatively charged uniformly by HEPES capping after synthesis (see Experimental section), as confirmed by $Z$-potential analysis, which shows values of $c a$. $-30 \mathrm{mV}$ (data not shown). Subsequently, MBNPs (44 pM final concentration) were incubated with the positively charged CV Raman reporter in a broad range of concentration, spanning from $8.27 \times 10^{-13} \mathrm{M}$ to $1.13 \times 10^{-5} \mathrm{M}$, i.e., largely below and above the theoretical saturation point of total surface coverage (CV $1.26 \times 10^{-7} \mathrm{M}$, see Experimental section). Fig. 5 reports representative SERS signals obtained for $\mathrm{CV}$ adsorbed onto these nanostructures. The observed vibrational modes of $\mathrm{CV}$ well agree with those reported in the literature, ${ }^{9,40}$ while no signals were detectable in the Raman spectra of bulk CV (10 mM, aqueous solution). Importantly, the Au MBNPs showed a higher Raman enhancement at all investigated CV concentrations with respect to that observed for the spherical NPs (0 mM HEPES). The spherical NPs presented a maximum in SERS intensities at a $\mathrm{CV}$ concentration of $4.2 \times 10^{-8}$, while the roughest MBNPs (25 mM HEPES) exhibit a maximum Raman enhancement at a CV concentration of $8.27 \times 10^{-13} \mathrm{M}$. In this case, the SERS intensity of rough MBNPs was $c a$. 2.5-fold higher than the best signal obtained with spherical NPs (Fig. 5). Noteworthy, we were able to attain such very high Raman signal with a reporter concentration which was five orders of magnitude lower than that giving the highest Raman signal for the spherical NPs. This behavior is partly due to the increasing molar extinction coefficient of the branched nanoparticles at the laser wavelength

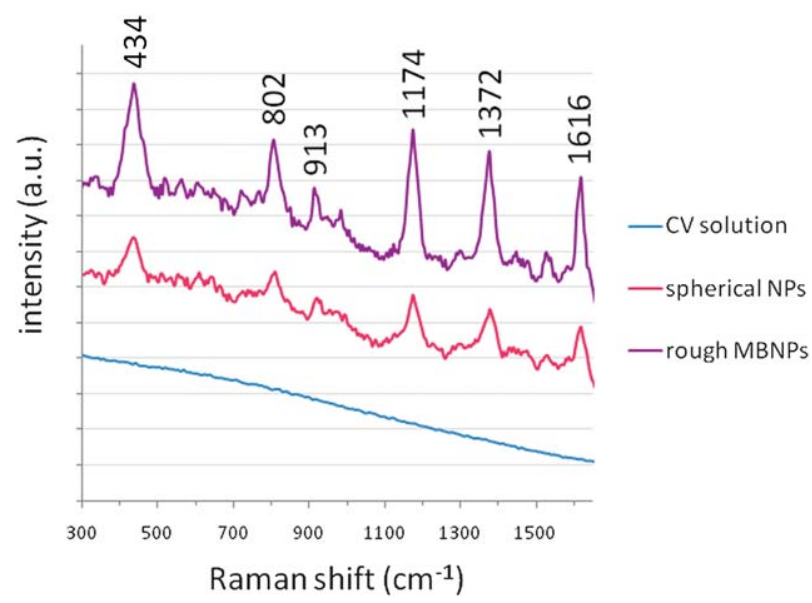

Fig. 5 SERS signals obtained for $\mathrm{CV}$ molecules adsorbed onto $60 \mathrm{~nm}$ spherical NPs (synthesized in $0 \mathrm{mM}$ HEPES) incubated with $4.2 \times$ $10^{-8} \mathrm{M}$ of $\mathrm{CV}$ and onto $60 \mathrm{~nm}$ rough MBNPs (synthesized in $25 \mathrm{mM}$ HEPES) incubated with $8.3 \times 10^{-13} \mathrm{M}$ of $\mathrm{CV}$. Blue line represents the Raman spectra of bulk CV (10 mM, aqueous solution). The observed vibrational modes of $\mathrm{CV}$ are highlighted.
(Table $\mathrm{S} 1 \dagger$ ); however, in the roughest particles most of the signal enhancement can be ascribed to the nanostructuration of these MBNPs that possesses very efficient SERS-active sites, with CV molecules preferentially adsorbing onto such sites. ${ }^{9}$ This enables an optimal detection of SERS modes at such low concentrations. In the case of MBNPs, the applied concentrations (both for $\mathrm{Au}$ MBNPs and for Raman reporter) were in the pM range, making the roughest MBNPs very attractive as SERS labels for biological imaging, and for chemical and bioanalytical sensing that requires high sensitivity and low detection limits.

\section{Conclusions}

In this work, we reported a novel seed-mediated synthetic route for the production of $\mathrm{Au}$ MBNPs that enables a precise and simultaneous control of both NPs size and surface nanostructuration. Such a result was achieved by combining the ability of $\mathrm{NH}_{2} \mathrm{OH}$ to act as a strong reducing agent only in the presence of gold surfaces (enlargement of gold seeds) with the property of HEPES to grow the seeds in an anisotropic way. The combination of $\mathrm{NH}_{2} \mathrm{OH}$ and HEPES allowed us to obtain high quality MBNPs, by altering the growth mechanism from aggregation based to anisotropic seeded growth. In particular, by tuning the HEPES concentration and using differently sized gold seeds, we were able to obtain increasing values of surface nanostructuration, resulting in the production of $\mathrm{Au}$ MBNPs with different final sizes (40 and $60 \mathrm{~nm}$, respectively). Such nanostructures were used to investigate their effects over the SERS efficiency of a Raman reporter (CV) adsorbed onto them. The Raman signal with the nanostructured gold NPs was demonstrated to be higher than that observed with reference spherical gold NPs, despite the use of a reporter concentration 5 orders of magnitude lower. This result suggests that MBNPs are promising candidates for biolabeling, imaging, biosensing, and therapeutic applications.

\section{Experimental}

We employed 18 and $40 \mathrm{~nm}$ spherical, citrate-capped gold nanoparticles, as seeds for the growing of bigger MBNPs. Gold seeds of $18 \mathrm{~nm}$ were prepared by the classical Turkevich-Frens method, ${ }^{41,42}$ using trisodium citrate as a reducing agent in aqueous solution. Briefly, $150 \mathrm{~mL}$ of $0.25 \mathrm{mM}$ aqueous solution of $\mathrm{HAuCl}_{4}$ (Sigma-Aldrich) was heated to boiling while stirring, then $1.15 \mathrm{~mL}$ of $0.1 \mathrm{M}$ aqueous solution of sodium citrate (Sigma-Aldrich) was added. The solution was kept gently boiling until a red wine color appeared and then slowly cooled down. Gold seeds of $c a$. $40 \mathrm{~nm}$ were prepared according to a two-step seed-mediated method proposed by Brown and Natan ${ }^{26}$ which allows the enlargement of $18 \mathrm{~nm}$ AuNPs (seeds) for the property of $\mathrm{NH}_{2} \mathrm{OH}$ to efficiently reduce $\mathrm{Au}^{3+}$ to bulk metal in the presence of $\mathrm{Au}$ surface. ${ }^{25}$ The synthesis was performed by adding $1.2 \mathrm{~mL}$ of aqueous $0.1 \mathrm{M}$ hydroxylamine sulfate (Sigma-Aldrich) and an appropriate amount of $18 \mathrm{~nm}$ gold seeds into $250 \mathrm{~mL}$ of water. The solution was kept under vigorous stirring and then $20 \mathrm{~mL}$ of $2 \mathrm{mM}$ aqueous solution of $\mathrm{HAuCl}_{4}$ was dropwise added to the seeds solution $\left(1 \mathrm{~mL} \mathrm{~min}^{-1}\right)$. After the addition of $\mathrm{HAuCl}_{4}$ solution was over, stirring was continued for $30 \mathrm{~min}$ and then sodium citrate was injected to a final concentration of $1 \mathrm{mM}$ to 
stabilize the colloidal solution. The quality of the so-prepared 18 and $40 \mathrm{~nm}$ seeds was verified by using TEM, UV-Vis spectroscopy, Dynamic Light Scattering (DLS) and $Z$-potential analysis (see Fig. S1†).

The synthesis of increasing nanorough $40 \mathrm{~nm}$ Au MBNPs was carried out in $25 \mathrm{~mL}$ aqueous solution presenting growing concentrations of HEPES (Sigma-Aldrich) adjusted to $\mathrm{pH} 7.0$ with $\mathrm{NaOH}$, namely $0.2,1,2.5,5$, and $25 \mathrm{mM}$. Then, the appropriate amount of $18 \mathrm{~nm}$ AuNPs (seeds) and $0.12 \mathrm{~mL}$ of $100 \mathrm{mM} \mathrm{NH} \mathrm{NH}_{2} \mathrm{OH}$ was added in the reaction mixture. The enlargement of $18 \mathrm{~nm}$ Au seeds was achieved by dropwise adding $5 \mathrm{~mL}$ of $0.8 \mathrm{mM}$ aqueous solution of $\mathrm{HAuCl}_{4}\left(0.5 \mathrm{~mL} \mathrm{~min}{ }^{-1}\right)$. The same enlargement procedure was employed to obtain $60 \mathrm{~nm}$ $\mathrm{Au}$ MBNPs, with the same trend of surface nanostructuration, employing the $40 \mathrm{~nm}$ AuNPs as seeds. These reactions were all conducted at $4{ }^{\circ} \mathrm{C}$. The quality of the MBNPs was investigated by TEM, HR-TEM, SEM, and UV-Vis spectroscopy.

For the SERS experiments, in a freshly prepared $60 \mathrm{~nm} \mathrm{Au}$ MBNPs series, HEPES solution ( $\mathrm{pH} 7.0$ ) was added to a final concentration of $25 \mathrm{mM}$ to ensure the same surface chemistry by HEPES capping. After stirring for 1 hour, nanoparticles were subjected to three washing steps by centrifugation and resuspension in ultrapure, sterile water. The nanoparticles series was investigated by DLS and $Z$-potential analysis to ensure good quality. The concentration of such Au MBNPs was determined as follows: the freshly synthesized Au MBNPs possess the same number of nanoparticles since we employed the same amount of $\mathrm{Au}$ seeds and chloroauric acid for all the synthesis regarding a specific final size. For this reason, the spectra shown in Fig. 3a and (b) represent the absorbance of solutions presenting the same number of nanoparticles, with increasing surface roughness. The molar extinction coefficient for spherical AuNPs is known ${ }^{43}$ and so, from the spectra obtained in Fig. 3, we extrapolated the theoretical molar extinction coefficient for the rough nanoparticles (from 0.2 mM HEPES to $25 \mathrm{mM}$ HEPES). By applying absorption spectroscopy, we diluted the negatively charged MBNPs to a final concentration of $0.044 \mathrm{nM}$. After, a reporter solution of Crystal Violet (CV) chloride (Sigma-Aldrich) was added to the colloidal solutions to a final concentration ranging from $8.27 \times 10^{-13} \mathrm{M}$ to $1.13 \times 10^{-5} \mathrm{M}$. The $\mathrm{CV}$ concentration needed to fully cover $0.044 \mathrm{nM}$ of $60 \mathrm{~nm}$ spherical gold NPs is calculated to be about $4.2 \times 10^{-8} \mathrm{M}$, considering that the surface area of a $\mathrm{CV}$ molecule is about $4 \mathrm{~nm}^{2}$ (assuming a parallel orientation of the adsorbed molecule at the NP surface). ${ }^{9}$ Prior to performing SERS experiments, the solutions containing SERS active nanostructures and Raman reporter, electrostatically interacting, were monitored by DLS in order to verify the absence of colloidal aggregations. SERS experiments on $\mathrm{Au}$ MBNPs were carried out by using a Jobin Yvon Fluorog 3 spectrofluorometer equipped with double monochromators and a cooled photomultiplier, and a $632.8 \mathrm{~nm}, 30.0 \mathrm{~mW} \mathrm{HeNe}$ laser as the excitation source.

\section{References}

1 C. Burda, X. Chen, R. Narayanan and M. A. El-Sayed, Chem. Rev., 2005, 105, 1025.

2 P. K. Jain, X. Huang, I. H. El-Sayed and M. A. El-Sayed, Acc. Chem. Res., 2008, 41, 1578.

3 B. K. Jena and C. R. Raj, Langmuir, 2007, 23, 4064.
4 K. Yu, K. L. Kelly, N. Sakai and T. Tatsuma, Langmuir, 2008, 24, 5849 .

5 T. R. Kuo, V. A. Hovhannisyan, Y. C. Chao, S. L. Chao, S. J. Chiang, S. J. Lin, C. Y. Dong and C. C. Chen, J. Am. Chem. Soc., 2010, 132, 14163.

6 J. E. Millstone, D. G. Georganopoulou, X. Xu, W. Wei, S. Li and C. A. Mirkin, Small, 2008, 4, 2176.

7 J. Chen, C. Glaus, R. Laforest, Q. Zhang, M. Yang, M. Gidding, M. J. Welch and Y. Xia, Small, 2010, 6, 811.

8 R. Huschka, O. Neumann, A. Barhoumi and N. J. Halas, Nano Lett., 2010, 10, 4117.

9 E. N. Esenturk and A. R. H. Walker, J. Raman Spectrosc., 2009, 40, 86.

10 J. Aaron, E. de la Rosa, K. Travis, N. Harrison, J. Burt, M. J. Yacaman and K. Sokolov, Opt. Express, 2008, 16, 2153.

11 S. K. Dondapati, T. K. Sau, C. Hrelescu, T. A. Klar, F. D. Stefani and J. Feldmann, ACS Nano, 2010, 4, 6318.

12 S. Wang, Z. Wu, F. Qu, S. Zhang, G. Shen and R. Yu, Biosens. Bioelectron., 2008, 24, 1020.

13 Z. Wang, J. Zhang, J. M. Ekman, P. J. A. Kenis and Y. Lu, Nano Lett., 2010, 10, 1886.

14 J. Xie, Z. Q. Zhang, J. Y. Lee and D. I. C. Wang, ACS Nano, 2008, 2, 2473.

15 P. S. Kumar, I. Pastoriza-Santos, B. Rodrìguez-Gonzàlez, F. J. Garcia de Abajo and L. M. Liz-Marzàn, Nanotechnology, 2008, 19, 015606.

16 O. M. Bakr, B. H. Wunsch and F. Stellacci, Chem. Mater., 2006, 18, 3297.

17 C. L. Nehl, H. Liao and J. H. Hafner, Nano Lett., 2006, 6, 683.

18 E. C. Hao, R. C. Bailey, G. G. Schatz, J. T. Hupp and S. Y. Li, Nano Lett., 2004, 4, 327.

19 J. R. Fernandez, A. M. Funston, J. P. Juste, R. A. A. Puebla, L. M. Liz-Marzan and P. Mulvaney, Phys. Chem. Chem. Phys., 2009, 11, 5909.

20 C. G. Khoury and T. J. Vo-Dinh, J. Phys. Chem. C, 2008, 112, 18849.

21 T. K. Sau and C. J. Murphy, J. Am. Chem. Soc., 2004, 126, 8648.

22 W. Wang, X. Yang and H. Cui, J. Phys. Chem. C, 2008, 112, 16348.

23 X. Q. Zou, E. B. Ying and S. J. Dong, Nanotechnology, 2006, 17, 4758.

24 G. Palui, S. Ray and A. Banerjee, J. Mater. Chem., 2009, 19, 3457.

25 G. Stremsdoerfer, H. Perrot, J. R. Martin and P. J. Clechet, J. Electrochem. Soc., 1988, 135, 2881.

26 K. R. Brown and M. J. Natan, Langmuir, 2008, 14, 726.

27 N. E. Good, G. D. Winget, W. Winter, T. N. Connolly, K. Izana and R. M. M. Singh, Biochemistry, 1966, 5, 467.

28 J. Xie, J. Y. Lee and D. I. C. Wang, Chem. Mater., 2007, 19, 2823.

29 S. Link and M. A. El-Sayed, J. Phys. Chem. B, 1999, 103, 8410.

30 F. Hao, C. L. Nehl, J. H. Hafner and P. Nordlander, Nano Lett., 2007, 7, 729

31 C. H. Kuo and M. H. Huang, Langmuir, 2005, 21, 2012.

32 J. L. Burt, J. L. Elechiguerra, J. Reyes-Gasga, J. M. MontejanoCarrizales and M. Jose-Yacaman, J. Cryst. Growth, 2005, 285, 681.

33 T. Vo-Dinh, H. N. Wang and J. Scaffidi, J. Biophotonics, 2010, 3, 89.

34 L. Fabris, M. Schierhorn, M. Moskovits and G. C. Bazan, Small, $2010,19,1550$.

35 X. M. Qian and S. M. Nie, Chem. Soc. Rev., 2008, 37, 912.

36 X. M. Qian, X. H. Peng, D. O. Ansari, Q. Yin-Goen, G. Z. Chen, D. M. Shin, L. Yang, A. N. Young, M. D. Wang and S. M. Nie, Nat. Biotechnol., 2008, 26, 83.

37 L. Rodríguez-Lorenzo, R. A. Alvarez-Puebla, I. Pastoriza-Santos, S. Mazzucco, O. Stephan, M. Kociak, L. M. Liz-Marzan and F. J. J. García de Abajo, J. Am. Chem. Soc., 2009, 131, 4616.

38 J. T. Krug, G. D. Wang, S. R. Emory and S. M. Nie, J. Am. Chem. Soc., 1999, 121, 9208.

39 M. Moskovits and D. H. Jeong, Chem. Phys. Lett., 2004, 397, 91.

40 D. Cialla, H. Uwe, H. Schneidewind, R. Moller and J. Popp, ChemPhysChem, 2008, 9, 758.

41 J. Turkevich, P. C. Stevenson and J. A. Hillier, Discuss. Faraday Soc., 1951, 11, 55.

42 G. Frens, Nature (London), Phys. Sci., 1973, 241, 20.

43 J. S. Lee, S. I. Stoeva and C. A. Mirkin, J. Am. Chem. Soc., 2006, 128, 8899. 